\title{
Reflets
}

Revue d'intervention sociale et communautaire

\section{Les mémoires de maîtrise en service social à l'Université d'Ottawa}

\author{
Alexandra Potvin, Aline Abdulnour, Benoit Veilleux, Carole Gagnon, Cindy \\ Lavoie, Denise Ouellette, Eliane Jeha, Geneviève Piché, Jean-Marie Mousenga, \\ Louise Provost, Magnolia Soutyrine, Marie-Ève Lavoie, Marie-Renée \\ Bernagène, Michèle Dumel, Rachel Hasan, Régis L. Luzolo, Sarah Poulin, Sonia \\ Richard, Stéphanie Pearce, Tania Duguay, Tania Paiement, Thomas Vanté et \\ Tina Delorme
}

Volume 9, numéro 1, printemps 2003

Le genre en contexte : pratiques sociales et représentations

URI : https://id.erudit.org/iderudit/010872ar

DOI : https://doi.org/10.7202/010872ar

Aller au sommaire du numéro

Éditeur(s)

Reflets : Revue ontaroise d'intervention sociale et communautaire

ISSN

1203-4576 (imprimé)

1712-8498 (numérique)

Découvrir la revue

Citer ce document

Potvin, A., Abdulnour, A., Veilleux, B., Gagnon, C., Lavoie, C., Ouellette, D., Jeha, E., Piché, G., Mousenga, J.-M., Provost, L., Soutyrine, M., Lavoie, M.-È., Bernagène, M.-R., Dumel, M., Hasan, R., Luzolo, R. L., Poulin, S., Richard, S., Pearce, S., Duguay, T., Paiement, T., Vanté, T. \& Delorme, T. (2003). Les mémoires de maîtrise en service social à l'Université d'Ottawa. Reflets, 9(1), 238-264. https://doi.org/10.7202/010872ar

Tous droits réservés (C) Reflets : Revue ontaroise d'intervention sociale et communautaire, 2002
Ce document est protégé par la loi sur le droit d'auteur. L'utilisation des services d'Érudit (y compris la reproduction) est assujettie à sa politique d'utilisation que vous pouvez consulter en ligne. 


\title{
Actions sociales : stratégies et contraintes dans le contexte socio-économique actuel
}

\author{
Alexandra Potvin \\ École de service social, Université d'Ottawa
}

Cette étude de cas a pour objectif d'évaluer une initiative communautaire d'intervention collective dans l'environnement socio-économique actuel. Pour ce faire, il s'agira d'abord de présenter la problématique en identifiant les politiques néo-libérales des gouvernements fédéral et provincial en matière des services sociaux, tout en exposant l'impact de cette intervention de l'État sur le bien-être collectif. Nous verrons que la municipalité d'Ottawa doit assumer des responsabilités financières accrues; que les agences communautaires doivent combler des besoins grandissants avec moins de ressources pécuniaires; et que les résidents doivent davantage surmonter des barrières sociales qui font décliner leur qualité de vie. Dans ce nouveau contexte de remodelage des politiques sociales et de compressions budgétaires, il devient pressant de contrecarrer les forces néo-libérales et d'influencer les administrations gouvernementales pour améliorer les conditions d'existence des classes défavorisées, ciblées et marginalisées par des politiques répressives. En s'inspirant d'une approche marxistestructurelle, un cadre d'analyse s'appuyant sur une perspective d'action sociale a été développé pour évaluer si le Groupe de travail sur la pauvreté à Ottawa a pu se faire entendre par les détenteurs de pouvoir. Des concepts clés, tels la conscientisation collective, la mobilisation, et l'empowerment, ont été mis en relief. Ce faisant, il est question d'exposer les tactiques revendicatives, les retombées ainsi que les difficultés rencontrées par le Groupe. Enfin, à l'aide de cette étude de cas, le présent travail vise à offrir des outils à la communauté en ce qui concerne les activités de revendication, de façon à inciter la renaissance de l'action sociale. 


\section{Entre deux cultures : stratégies utilisées par les jeunes canadiennes libanaises pour faire face aux conflits intergénérationnels}

Aline Abdulnour

École de service social, Université d'Ottawa

Cette étude explore les stratégies mises de l'avant par les jeunes Libanaises nées ou ayant émigré au Canada, pour faire face aux conflits intergénérationnels dans leurs familles. Nous nous intéressons plus particulièrement, aux forces et aux compétences déployées par les jeunes dans ces situations. Tout en explorant la nature de ces conflits au sein de la famille immigrante, cette recherche vise d'abord à cibler les stratégies qui permettent aux adolescentes de traverser les difficultés en continuant de se développer.

Nous avons privilégié une méthodologie qualitative qui a permis d'explorer la réalité vécue par les jeunes et de la mettre en avant plan. En tout, six participantes ont choisi de faire partie de la recherche. Deux groupes focus ont été formés avec trois participantes dans chacun.

L'analyse des propos des participantes a fait émerger trois thèmes principaux. Le premier, le projet d'immigration des parents, est un thème central puisque celui-ci a une influence sur les relations intergénérationnelles, sur les conflits qui surgissent entre les membres de la famille, qui influencent à leur tour la recherche d'identité des jeunes adolescents et adolescentes. Le deuxième thème porte sur les tensions vécues par les jeunes, notamment dans leur quête d'identité. Le dernier traite des stratégies utilisées par les jeunes pour surmonter les difficultés.

Finalement, nous avons dégagé quelques pistes de réflexion pour l'intervention sociale chez les jeunes qui vivent des tensions intergénérationnelles. Permettre aux jeunes de s'exprimer sur leur 
vécu et de partager leurs expériences, leur a permis de s'affirmer comme acteurs sociaux de leur situation.

\title{
La puissance, l'impuissance et la douleur dans la vie des hommes
}

\author{
Benoit Veilleux \\ École de service social, Université d'Ottawa
}

Dans un monde dominé par les hommes, le monde des hommes est par définition un monde où l'enjeu principal est le pouvoir» (Kaufman 1994). Ce pouvoir est omniprésent au sein de notre société. C'est à travers les différentes institutions telles que la famille, la religion, la politique et l'économie, entre autres, que l'homme exerce son pouvoir et son contrôle sur l'ensemble du système. Mais sur le plan individuel, la relation que l'homme a développé avec le pouvoir est sans contredit fort différente. Peu d'hommes peuvent prétendre se sentir bien en contrôle de leur vie. Si certains peuvent jouir d'un statut privilégié, la grande majorité des hommes vivent des contradictions importantes à l'égard du pouvoir. Dans leurs relations personnelles, leurs emplois et autres, contrairement à la croyance populaire, un pourcentage important d'hommes n'est pas toujours en position de force.

Ce mémoire propose une réflexion à l'égard du mode de socialisation de l'homme et de certaines des conséquences de cette socialisation sur sa vie quotidienne. En d'autres mots, si l'homme occupe une place privilégiée au sein de notre société comment expliquer qu'il présente une espérance de vie moins élevée que celle de la femme? Pourquoi est-il plus susceptible de souffrir du cancer? Comment expliquer que l'homme est plus sujet à des maladies du cœur? Pourquoi y a-t-il plus de morts par suicide chez l'homme que chez la femme? En somme, est-ce que le pouvoir recèle des contradictions, des conséquences qui en bout de ligne étouffent l'homme moderne? 
Ce travail présente trois grands thèmes. D'abord, il offre une revue des différents passages de l'histoire de notre société qui ont influencé la construction sociale de l'homme. Cette partie est la pierre angulaire du mémoire puisqu'elle permet de situer l'homme dans l'histoire de notre société à partir d'une réflexion sur le patriarcat. Dans un deuxième temps, ce travail met en relief les contradictions et l'illusion qui entourent la relation de l'homme avec le pouvoir. Cette section met également l'accent sur la douleur et l'aliénation qui résultent du mode de socialisation de l'homme dans notre société. Enfin, ce mémoire dépeint et critique la situation des hommes dans le contexte actuel. Dans cette même foulée, cette section présente les différents enjeux et défis auxquels sont confrontés les hommes en ce début de millénaire.

\title{
« Ça s'peut pas... ça fait pas d'sens ». Le harcèlement moral en milieu de travail : une question de stratégies
}

\author{
Carole Gagnon \\ École de service social, Université d'Ottawa
}

Le harcèlement moral est un problème très répandu dans le milieu du travail, selon les témoignages de plusieurs travailleurs et travailleuses. Les effets sur les victimes se répercutent à plusieurs niveaux, y compris la santé physique et psychologique, l'estime de soi, les relations familiales et le risque de suicide, entre autres. Les victimes doivent donc recourir à diverses stratégies d'ajustement pour contrer les effets néfastes de cette violence. Par contre, très peu de scientifiques se sont intéressés à cet aspect de la problématique. Le but de cette étude est d'examiner le vécu des victimes de harcèlement en milieu de travail en portant une attention particulière aux stratégies d'ajustement auxquelles elles ont recours. Empruntant un devis de recherche qualitative exploratoire, nous avons procédé à l'entrevue semi-dirigée de quatre femmes et de deux hommes qui ont accepté de partager leurs expériences par rapport à leur victimisation au travail. 
Nos résultats indiquent que les victimes de harcèlement en milieu de travail traversent trois phases de transition : "Ça s'peut pas... j'ai dû avoir mal compris "; «Y'a pas l'droit de m'faire ça! »; et "Faut que j'décroche ". Durant ces étapes, les victimes démontrent une adaptabilité remarquable aux conditions instables caractéristiques du processus de victimisation. Nos données indiquent que les victimes ont davantage recours à diverses stratégies actives et directes axées sur la résolution du problème durant les deux premières phases mais se penchent plutôt sur des stratégies passives qui misent sur la réduction des symptômes durant la dernière étape. Cette flexibilité d'ajuster le choix de stratégies aux nombreux obstacles affrontés fait preuve de la résilience, la persévérance et la créativité des victimes. Cette étude introduit des pistes de réflexion au niveau des politiques sociales, de l'intervention et de la recherche par la mise en évidence des expériences, des forces et des besoins des victimes de harcèlement en milieu de travail.

\section{Le rôle du sentiment amoureux dans les relations amoureuses et sexuelles à l'adolescence}

Cindy Lavoie
École de service social, Université d'Ottawa

Le but de ce mémoire est de montrer l'influence du sentiment amoureux dans les conduites sexuelles des adolescentes et adolescents. La méthodologie choisie consiste en une recherche sur documents dans le but d'analyser l'éventail des ouvrages qui ont traité du sujet. L'analyse tente donc de mieux cerner le lien entre l'amour que les adolescentes et les adolescents éprouvent pour un partenaire et le comportement sexuel qu'ils adoptent avec lui. Plus encore, nous cherchons à savoir si les garçons et les filles agissent de la même façon et s'ils ont des opinions qui se rapprochent en regard du sentiment amoureux. Le mémoire 
permet ainsi d'arriver à une meilleure compréhension des comportements à l'égard de la sexualité à l'adolescence.

\section{Protéger les enfants... pour un meilleur avenir : les enjeux actuels de la protection sociale en Ontario}

\section{Denise Ouellette}

École de service social, Université d'Ottawa

L'objectif de ce mémoire est de faire un bilan documentaire analysant les enjeux liés aux innovations dans le domaine de la protection de l'enfance, soit les changements apportés depuis la réforme de la protection de l'enfance de 1997 en Ontario. Nous privilégions celles qui ont un impact direct sur la pratique sociale, soit la Loi sur les services à l'enfance et à la famille (LSEF), le modèle d'évaluation des risques et le modèle de Looking after Children (LAC). Le concept de la diffusion de l'innovation en agence de protection de l'enfance comme cadre théorique de ce mémoire nous permettra de jeter un meilleur éclairage sur les enjeux actuels des changements implantés dans les Sociétés de l'aide à l'enfance. Trois méthodes de repérage ont été utilisées pour localiser les publications présentées dans l'analyse subséquente.

Depuis le début de la réforme ontarienne, nous avons constaté une augmentation du nombre d'enquêtes et d'enfants pris en charge. Nous percevons une primauté du légal dans le processus décisionnel et dans les actions des intervenantes ou intervenants. Des questions sont soulevées quant à la quantité et aux conditions de travail présentes dans les agences de protection. En plus d'intervenir auprès des enfants les plus vulnérables de la société, les intervenantes sociales et les intervenants sociaux sont constamment avertis que leurs prises de décisions dans la gestion de cas pourraient avoir un effet sur leur crédibilité, voire résulter en une poursuite judiciaire. Le stress encouru par les intervenantes 
sociales et les intervenants sociaux augmente. On ne sollicite pas leur avis sur les enjeux actuels; c'est pourtant un concept clé dans la diffusion des innovations.

Nous devons collaborer, former des partenariats entre les divers systèmes de notre structure sociale afin de diriger notre énergie vers la protection de l'avenir de nos enfants d'aujourd'hui!

\section{L'intervention interculturelle avec les familles immigrantes dans un contexte de bien-être à l'enfance : perceptions et défis des intervenants}

Eliane Jeha
École de service social, Université d'Ottawa

Cette recherche qualitative de type exploratoire aborde l'expérience des travailleuses sociales de la Société de l'aide à l'enfance (SAE) qui interviennent auprès des familles immigrantes. La recherche a pour objectif d'identifier certains des principes d'intervention qui guident les travailleuses sociales dans un contexte de bien-être à l'enfance avec des familles immigrantes et les stratégies qu'elles privilégient pour surmonter les difficultés rencontrées. Nous avons eu recours à des entrevues semi-dirigées avec cinq intervenantes qui travaillent à la SAE pour explorer avec elles leur expérience à l'égard de nos questions de recherche.

L'analyse des résultats a permis de dégager quatre principaux thèmes. Dans un premier temps, les répondantes ont souligné plusieurs défis spécifiques au travail en contexte d'autorité interculturel tels que les barrières linguistiques, certains enjeux liés au pouvoir et au rôle des intervenantes, ainsi que des enjeux émanant des transformations des rôles et du pouvoir au sein de la famille immigrante provoquées par le processus d'immigration. Un deuxième thème apporte un éclairage sur certains des principes 
qui animent l'intervention visant à créer un espace de travail interculturel et un troisième, sur les rôles assumés par les répondantes, notamment sur les plans de l'éducation et du soutien aux parents. L'analyse se termine par l'exploration des stratégies mises en place pour surmonter les défis rencontrés lors des interventions auprès des familles immigrantes.

\section{La surdité chez 5 adolescents et adolescentes : communication, insertion et solutions}

\section{Geneviève Piché}

École de service social, Université d'Ottawa

Ce mémoire rend compte d'une étude dont l'objectif premier consistait à explorer l'impact de la surdité sur l'insertion sociale des jeunes sourds. Peu de recherches ont été faites sur les relations sociales des jeunes sourds francophones et il existe encore moins d'études qualitatives basées sur leurs perspectives. Cette étude nous permet d'avoir un aperçu des enjeux auxquels font face les jeunes sourds dans divers milieux sociaux et de connaître leurs points de vue face aux défis qu'ils rencontrent.

Notre cueillette de données s'est réalisée par le biais d'entrevues semi-dirigées auprès de cinq jeunes sourds âgés de 16 à 21 ans. Les résultats montrent que la surdité affecte les interactions sociales des jeunes sourds. En effet, ces jeunes rencontrent souvent des bris de communication dans certains contextes sociaux, ce qui les amène à se sentir isolés et incompris. Par ailleurs, les jeunes sourds gestuels (communiquant en langage des signes) et oralistes (parlant le français) interrogés vivent ces enjeux différemment. Les oralistes rencontrent plus d'obstacles à l'école et dans leur réseau d'amis tandis que les gestuels se sentent plus isolés dans leur famille. D'autre part, la plupart des répondantes et répondants ont de bons réseaux de soutien et d'excellentes stratégies face aux difficultés qu'ils rencontrent. 
Nous concluons que la surdité n'amène pas le jeune à se sentir exclu, mais que certains facteurs, tels les bris de communication, les préjugés, l'ignorance et le manque de services adéquats entraînent des sentiments de tristesse, de frustration ou d'isolement. Cependant, des éléments tels que le soutien de personnes importantes à leurs yeux, l'implication dans différentes activités sociales, le lien avec d'autres personnes sourdes et l'utilisation de ressources personnelles permettent d'atténuer l'impact de ces difficultés.

\section{La crise du logement à Ottawa et les ménages à faible revenu}

Jean-Marie Mousenga

École de service social, Université d'Ottawa

Après une longue période progressiste mettant en scène de nombreux programmes d'aide aux personnes dans le besoin à partir de 1984, le gouvernement fédéral axa ses priorités ailleurs que dans le domaine des services sociaux. Ces politiques ont eu pour effet de créer des inégalités entre les riches et les pauvres. Dans ces conditions, il devenait normal d'accorder une plus grande attention au problème crucial que vivaient les ménages à faible revenu suite à la crise du logement conséquent à l'abandon par le gouvernement fédéral de ses politiques sociales.

Notre étude, qui se veut exploratoire, vise à mieux connaitre les causes de la crise du logement qui sévit à Ottawa en comparaison de celles de Montréal, de Toronto et de Vancouver.

La question de cette recherche est la suivante : considérant que selon une opinion généralement admise, l'État doit apporter son aide aux personnes et aux familles qui éprouvent des difficultés économiques par l'entremise de politiques et interventions sociales adaptées à leurs besoins et devant la crise du logement qui sévit 
dans les grandes villes canadiennes et principalement à Ottawa, est-ce que le gouvernement fédéral a mis en place des moyens permettant de répondre à la demande grandissante de logements sociaux destinés aux ménages à faible revenu? Si oui, ces moyens répondent-ils aux besoins et aux attentes de ces ménages?

Quoique la question posée s'adressait préalablement au gouvernement fédéral dans la période qui suivait les mesures de compressions budgétaires, notre recherche nous a amené à constater que les deux autres paliers de gouvernements se font complices de ce choix de politiques, en emboîtant le pas au gouvernement fédéral. En effet, profitant des politiques de restriction en vigueur à Ottawa, le gouvernement de l'Ontario et la Ville d'Ottawa orientaient aussi leur politique dans la même direction, aggravant ainsi une situation déjà désastreuse tout en se renvoyant la balle les uns aux autres.

Les principaux résultats obtenus suite à la compilation des données (les caractéristiques socio-économiques, le revenu, les dépenses en besoins essentiels, le taux d'inoccupation, le coût mensuel des loyers et l'indice des prix à la consommation) montrent dans une large mesure que l'absence de politiques de la part des trois paliers de gouvernements est à la base de cette crise et confirment donc l'objectif de cette recherche. Le résultat est que les ménages à faible revenu ont vu leurs difficultés s'amplifier, ce qui les amena entre autres à accorder une part de plus en plus importante au règlement du loyer, négligeant du même coup les dépenses liées aux besoins essentiels. 


\title{
Les trajectoires de jeunes adultes en situation précaire. L'organisation des processus d'insertion sociale et professionnelle
}

\author{
Louise Provost \\ Université d'Ottawa, École de service social
}

Ce mémoire fait l'examen de parcours individuels et de cheminements de jeunes adultes à faible revenu résidant de manière temporaire dans une ressource d'hébergement. Nous avons examiné les situations de précarité de ces jeunes non comme une finalité mais comme des éléments d'un parcours de vie qui n'en sont pas nécessairement les déterminants principaux. Cette étude nous permet de cerner la perception de jeunes précaires au sujet des éléments personnels et structurels qui viennent appuyer ou non leur insertion sociale et professionnelle et d'explorer les stratégies déployées en faveur de leur insertion sociale et professionnelle.

Nous avons privilégié l'analyse de contenu de données recueillies à travers des entrevues semi-dirigées effectuées auprès de trois jeunes hommes et trois jeunes femmes âgés entre 20 et 28 ans. Les entrevues ont été réalisées dans le cadre d'une recherche évaluative du Réseau des Petites Avenues (RPA) (Charbonneau et Molgat : 2002). Après avoir identifié les étapes qui jalonnent les parcours de ces jeunes vers le monde adulte, force nous est de reconnaître que le contexte de précarité dans lequel ils vivent ne les condamne pas à une vie marginale. Au contraire, le passage dans la ressource leur permet d'identifier les stratégies nécessaires pour organiser une transition vers une insertion et une participation sociale et professionnelle plus complètes.

Nous concluons que bien que les différents facteurs individuels et structurels, inscrits dans le parcours (organisation familiale, isolement relationnel, manque d'argent, difficulté de gestion des finances personnelles et cercles vicieux du travail atypique) 
affectent les passages à la vie adulte, ceux-ci ne sont pas des déterminants immuables de l'insertion sociale et professionnelle. Bien que ces facteurs balisent les parcours, les jeunes ne sont pas "victimes" des circonstances, mais sont des "sujets " capables d'agir sur leur vie et de modifier leurs trajectoires. L'analyse des discours a ainsi mis en perspective les actions entreprises par les répondants et répondantes pour accéder à une meilleure qualité de vie. C'est à travers l'espace d'exploration que permet leur séjour au Réseau des Petites Avenues que plusieurs d'entre eux expérimentent des avenues ou voies qu'ils n'auraient pu envisager dans un autre contexte. C'est par cette expérimentation que les jeunes réussissent à bâtir leur identité, leur autonomie et leur indépendance. La recherche et l'intervention sont interpellées par cette perspective d'analyse et ces conclusions de recherche.

\section{L'économie sociale : une nouvelle forme de régulation de la société dans un contexte de mutations?}

\section{Magnolia Soutyrine}

École de service social, Université d'Ottawa

L'économie sociale, nommée aussi tiers secteur, est vue par beaucoup comme une solution aux problèmes sociaux contemporains tels que la crise de l'État-providence et celle de l'emploi. Dans une recension des écrits sur l'économie sociale afin d'analyser son potentiel et son implication pour l'intervention sociale, nous avons trouvé que, en effet, celle-ci peut apporter des solutions. Cependant, elle comporte aussi des pièges. Les activités issues de l'économie sociale sont classées en trois grandes branches : les coopératives, les associations et les mutuels. Malgré leur diversité, elles gardent les même principes fondamentaux : le service, l'autonomie, la démocratie et la primauté des personnes et du 
travail sur le capital. Ces postulats peuvent être la base d'un renouvellement des pratiques socio-économiques, où l'argent est au service de l'humanité et non pas le contraire, et où les relations humaines se bâtissent démocratiquement. Ainsi, il se peut que les salaires offerts soient inférieurs à ceux offerts par les entreprises privées et étatiques, mais la satisfaction au travail peut être supérieure.

L'économie sociale contribue non seulement à la création d'emplois et à la cohésion sociale, mais aussi à la création des espaces d'autonomie, de démocratie, de réflexion et de participation citoyenne, où la collectivité peut identifier des besoins et proposer des solutions. Nous trouvons également que la logique de l'économie sociale, étant différente de celle du marché, est basée sur le bénévolat, la réciprocité, l'entraide et le don, éléments nécessaires pour le maintien du lien social.

C'est ainsi que les intervenantes et intervenants sociaux, surtout du milieu communautaire, ont tendance depuis une décennie à favoriser une approche plus globale, visant le développement social et économique des communautés. Cette approche conjugue l'éducation, la santé et les services sociaux, la formation de la main-d'oeuvre et le développement local.

\title{
Les intervenants sociaux face aux besoins des familles qui ont adopté à l'extérieur du pays
}

\author{
Marie-Ève Lavoie \\ École de service social, Université d'Ottawa
}

Cette recherche porte sur les défis que doivent affronter des familles issues de l'adoption internationale et ce, selon les perceptions d'intervenantes en service social qui, dans leur travail, ont rencontré des familles adoptives faisant face à des difficultés. La question de départ de l'étude se pose ainsi : comment, dans les 
pratiques du service social, les difficultés vécues par les familles qui ont adopté un enfant hors du pays sont-elles abordées? Pour répondre à cette question, nous avons interrogé cinq intervenantes sur leurs perceptions des difficultés qu'elles rencontrent en consultation. À cette fin, nous avons utilisé un guide d'entrevue semi-dirigée. Le recrutement est de type non probabiliste intentionnel, soit grâce à un échantillon typique et un échantillon obtenu par effet boule de neige auprès d'intervenantes et de personnes impliquées dans le milieu de l'adoption internationale.

Trois thèmes principaux émergent de l'analyse des données. Dans un premier temps, les intervenantes sociales offrent leur perception sur les difficultés des familles adoptives, soulignant l'attachement, le deuil du passé et l'identité comme étant des défis importants pour les familles. Les participantes relèvent également des défis au niveau du mésosystème des familles, défis issus notamment du manque de ressources pour les familles en besoin. Les stratégies d'intervention auprès des parents, du réseau familial et des intervenantes elles-mêmes font l'objet de notre troisième thème. Finalement, l'analyse permet de dégager quelques pistes de réflexion pour l'intervention sociale dans le domaine de la postadoption.

\title{
Familles haïtiennes dans la société québécoise : facteurs d'intégration et approches d'intervention
}

\author{
Marie-Renée Bernagène \\ École de service social, Université d'Ottawa
}

Depuis environ 40 ans, le Québec fait face à une nouvelle réalité, soit la présence de milliers de familles d'origine haïtienne sur son territoire. L'expansion rapide qu'a connue l'immigration des familles haitiennes au Québec a aussi accéléré l'ampleur et le nombre des problèmes qui surgissent au sein de cette communauté. 
La présente étude a pour but d'identifier et de décrire les différents facteurs qui font obstacle à l'intégration des familles haïtiennes au Québec. Il s'agit d'une part, de cerner les réalités des familles haïtiennes qui ont le plus de difficulté à s'insérer dans la vie québécoise, sur les plans économique et affectif, et d'autre part, de comprendre les caractéristiques propres à la communauté haïtienne par leurs valeurs et leurs schèmes culturels afin d'identifier leurs besoins et leurs stratégies pour faire face à ces difficultés. Il est vrai que l'expérience de l'immigration présente un nombre de caractéristiques communes, mais la communauté haïtienne a néanmoins ses particularités qui font d'elle une communauté unique.

Cette étude se base sur une recherche documentaire qui trace le portrait de la communauté haïtienne au Québec. Un profil des ressources sur lesquelles ces familles peuvent compter a été élaboré. Elle présente également certaines pistes d'intervention pouvant éclairer et favoriser une prise de conscience chez les intervenants afin de les rendre plus attentifs à la culture des familles haïtiennes et de comprendre ces familles et les difficultés auxquelles elles font face dans leur quotidien au Québec.

\section{Groupes racisés et citoyenneté au Canada : un défi pour le travail social}

\section{Michèle Dumel \\ École de service social, Université d'Ottawa}

Depuis plus d'une vingtaine d'années, le Canada est un pays pluriethnique et doit, de ce fait, répondre aux enjeux liés à la différence culturelle et au racisme. Pourtant, dans sa vision libérale de la citoyenneté, basée sur des principes d'égalité formelle, il ne permet qu'une reconnaissance limitée de la différence. De plus, il accuse un passé de mesures et de politiques discriminatoires envers les populations racisées qui aujourd'hui encore, continuent à en 
subir les effets néfastes. Ce mémoire cherche à savoir quels effets ces enjeux ont sur la citoyenneté des membres des groupes racisés au Canada et quel rôle le service social peut jouer pour favoriser l'accès de ces groupes à une citoyenneté pleine et entière.

Nous examinerons ici les principales théories de la citoyenneté dans les sociétés occidentales, particulièrement celle qui prévaut au Canada. Nous chercherons ensuite à évaluer la citoyenneté des groupes racisés au moyen d'une étude de leur situation socioéconomique. D'après certains théoriciens (Kymlicka 1992, 1995, 2001, Taylor 1994), les désavantages sociaux, notamment la pauvreté, sont soulignés comme étant des obstacles à une citoyenneté pleine et entière. En effet, selon Taylor (1994), les gens que la pauvreté empêche de participer à la vie sociale sont relégués à un statut de "citoyens de seconde classe ". Cette étude est aussi une réflexion sur le rôle du travail social en ce qui a trait à la question de la citoyenneté des groupes racisés. Le travail social a traditionnellement négligé les diverses dimensions de la discrimination raciale dans son analyse des problèmes sociaux. Cette réflexion met de l'avant des perspectives liées à l'intervention dans ce domaine.

\title{
«Le plus gros là dedans, c'est qu'il faut pas que tu aies honte quand tu fais le mieux que tu peux (111) ».
}

\author{
Rachel Hasan \\ École de service social, Université d'Ottawa
}

Le phénomène de l'intervention précoce en matière de violence chez les jeunes enfants préoccupe non seulement la société mais aussi le gouvernement ontarien, notamment par l'introduction de la Loi sur la sécurité dans les écoles. L'étude suivante décrit 23 enfants manifestant des conduites violentes, la famille et ses enjeux et la perception des parents à l'égard de l'apport de l'école. Il s'agit d'un sous-échantillon d'un groupe de 70 enfants participant 
au projet de recherche Pour contrer la violence chez les 3 à 9 ans : une collaboration entre les écoles et les parents concernés, occasion d'empowerment pour les parents. Pour que les parents puissent participer à cette recherche, leurs enfants devaient poser des gestes violents à l'égard de leurs pairs de façon fréquente et stable, et être l'objet d'une suspension scolaire ou d'une possibilité de suspension.

Les parents interrogés ont participé à des entrevues structurées ou semi-structurées. Ils sont presque tous d'origine canadiennefrançaise, au-dessus du seuil de la pauvreté et recevant de l'aide d'une professionnelle ou d'un professionnel. La majorité des enfants sont des garçons. Les résultats de cette étude montrent que les conduites violentes sont souvent en parallèle avec des états de dépression et d'anxiété chez les enfants ainsi qu'avec le rejet des pairs à l'école. Il semble que les pratiques parentales ne sont pas uniquement la source du problème, mais plutôt les conflits conjugaux, le manque de temps de qualité à offrir à l'enfant et l'absence du père. La suspension scolaire externe n'est pas appréciée par les parents. L'étude suggère qu'un soutien social qui leur serait approprié permettrait d'aider à contrer les conduites violentes des enfants en question.

\title{
Les défis de l'intervention féministe auprès des femmes violentées en provenance $d^{\prime}$ 'Afrique subsaharienne
}

\author{
Régis L. Luzolo \\ École de service social, Université d'Ottawa
}

La violence conjugale est devenue un problème social dans notre société.Au Canada, une femme sur six est victime de violence de la part de son partenaire et cette estimation est jugée conservatrice par les experts (Statistique Canada 1998). Cette problématique 
est particulière dans la mesure où elle soulève la question très sensible des rapports sociaux entre les sexes. Elle entraine des souffrances physiques et morales et nous interpelle comme individu sur le droit élémentaire à la sécurité de la personne.Avec l'immigration, les intervenantes et les intervenants rencontrent de plus en plus de clientes provenant des pays lointains et des cultures différentes de la leur. L'arrivée de cette nouvelle clientèle pose des défis à l'intervention traditionnelle.

Même si la littérature met en évidence les parallèles et les constantes dans la condition des femmes à travers le monde : violence, pauvreté, position de subordination dans le quotidien et dans l'organisation sociale et politique, il n'en demeure pas moins vrai que les conditions spécifiques dans lesquelles ces constantes s'incarnent varient selon le milieu. De fait, la situation des femmes africaines et d'autres femmes de couleur qui ont immigré au Canada est plus complexe, car elles subissent de multiples oppressions à cause de la couleur de leur peau, de leur culture, de leur religion et de leur statut; cette situation particulière appelle à une intervention plus centrée sur leur réalité.

\section{Un chez-soi pour les personnes psychiatrisées : la situation en Outaouais et à Ottawa}

\section{Sarah Poulin}

École de service social, Université d'Ottawa

Avoir un chez-soi est une condition de l'identité sociale. En Amérique du Nord, avoir un endroit où habiter est une condition fondamentale à une bonne santé physique et émotionnelle. Par contre, les personnes psychiatrisées n'ont pas toujours ce droit au logement.

Depuis le début de l'histoire, la personne psychiatrisée vit différentes formes d'exclusion, incluant celle de l'habitation. Elle 
a été mise en prison, en asile, dans des hôpitaux. Au Québec comme en Ontario, les politiques voulaient désinstitutionnaliser les personnes afin qu'elles puissent vivre au sein de la collectivité. Par contre, avec un manque de planification et de mesures adéquates, ces gens ont vécu beaucoup d'échecs dans la communauté qui a été mal préparée pour les recevoir. Des foyers de groupe et des familles d'accueil ont été développés pour répondre à ce besoin pressant. Cependant, ces modèles qui devaient être plus humains n'ont su que reproduire l'institution.

Au fil des années, les politiques tiennent compte davantage du choix de la personne et veulent assurer une qualité de vie plus adéquate. De là on voit une réorientation des politiques favorisant le soutien au logement qui consiste à intégrer les personnes psychiatrisées dans un logement "normal » avec l'appui du réseau de la santé.

Ce mémoire examine la mise en place de la réorientation de la politique de chaque côté de la frontière Ontario/Québec, plus précisément d'Ottawa et de l'Outaouais. Nous tentons par là de voir les différences et les ressemblances entre ces deux milieux où s'effectue la pratique du travail social dans une région frontalière et ce, en regard des conditions de pratique d'une telle politique et de son potentiel d'inclusion sociale pour une population qui constitue une des figures contemporaines de l'exclusion.

\section{Vivre en famille : la perception des enfants biologiques de parents d'accueil}

Sonia Richard
École de service social, Université d'Ottawa

Depuis les années 1930, le placement des enfants dans des familles d'accueil est le moyen privilégié pour les protéger de situations d'abus, de négligence et d'abandon. Plusieurs études se sont 
intéressées aux effets du placement sur les enfants placés, mais très peu d'entre elles ont exploré l'impact sur les foyers substituts et sur les enfants biologiques de ces derniers. Notre étude examine la perception qu'ont les enfants biologiques de familles d'accueil $\mathrm{du}$ fonctionnement de leur famille ainsi que leur adaptation, et identifie les ressources qui leur ont été disponibles. Des entrevues semi-dirigées ont été menées auprès de trois enfants biologiques francophones de famille d'accueil, recrutés via une agence de protection à l'enfance de l'est de l'Ontario.

Les participants ont indiqué des effets positifs de la garde nourricière, tels le rapprochement des membres de leur famille, un développement psychologique et émotionnel, et l'éveil d'un sentiment de valorisation personnelle.Toutefois, des effets négatifs ont aussi été soulignés par certains participants. Entre autres, ceuxci ont indiqué que la garde nourricière pouvait engendrer une distance entre les membres de leur famille, et qu'elle pouvait aboutir à un sentiment de manque d'attention venant de leurs parents. S'il y a eu des expériences similaires entre nos participants, il semble exister aussi des différences reliées à l'origine culturelle, aux types de familles d'accueil (relèves versus conventionnelles), aux structures familiales, à l'âge des enfants biologiques et de ceux placés, et aux stratégies d'adaptation développées.

Nos résultats indiquent quelques facteurs pouvant favoriser une bonne adaptation. Selon les participants, une bonne communication au sein de la famille serait un élément facilitant la transition des enfants biologiques. Aussi, des périodes de répit semblent rassurer ces enfants sur leur importance au sein de leur famille. De plus, nos données soulignent l'importance d'un soutien de la part des travailleuses et des travailleurs sociaux, en vue de bien préparer les enfants biologiques à envisager les implications négatives de la garde nourricière. Ainsi, il est primordial pour ces intervenantes et intervenants de continuer à s'informer des effets de la garde sur chaque membre de la famille substitut.

Il serait opportun que d'autres recherches étudient l'impact des ethnies et des divers types de compositions familiales sur la dynamique des familles d'accueil, et sur le vécu de chacun de ses 
membres. De plus, des recherches comparatives pourraient examiner l'expérience des membres de divers types de familles d'accueil, ainsi que le point de vue de différentes personnes impliquées. Nous espérons que notre étude contribuera à une meilleure compréhension des effets et des conséquences de la garde nourricière sur le fonctionnement des familles d'accueil, et de ses implications, immédiates et futures, sur les enfants biologiques de parents nourriciers.

\section{Vivre avec le DAH : La perception des mères franco- ontariennes sur le soutien et sur la qualité de rôle maternel}

Stéphanie Pearce
Université d'Ottawa, École de service social

Dans notre société, la responsabilité des soins d'un enfant atteint d'un handicap repose surtout sur la mère, donc elle assume la plupart des coûts associés à cette tâche. Il en est de même pour les mères d'enfants ayant un handicap invisible comme le déficit d'attention avec hyperactivité (DAH). La plupart des recherches dans le domaine se penchent sur les questions reliées à la gestion médicale du DAH et de ses symptômes ou sur l'impact de ceuxci sur la famille, sans étudier les facteurs environnementaux tels le soutien. Les études portant sur des mères franco-ontariennes d'enfants atteints du DAH sont presque inexistantes.

Notre mémoire s'est réalisé dans le cadre d'une étude qualitative auprès de 40 mères d'Ottawa qui ont un emploi et qui s'occupent d'un enfant de 6-17 ans avec le DAH (Home et Pearce, 2002). Cette étude explore la qualité de rôle maternel (gratifications et difficultés) ainsi que les sources et les types de soutiens disponibles qui peuvent contribuer à améliorer la qualité de ce rôle. Nous avons recruté les mères par le biais des services 
pour enfants et familles, dans des endroits publics, et par l'intermédiaire de deux groupes d'entraide qui s'intéressent au DAH. Notre échantillon spécifique comporte 11 mères francophones. La plupart d'entre elles $(70 \%)$ vivent avec plus d'un membre de la famille atteint du DAH et plusieurs enfants (75\%) cumulent d'autres désordres ou handicaps.

Les données de nos entrevues semi-dirigées ont été soumises à une analyse de contenu thématique. Les résultats indiquent que ces mères rencontrent plus de difficultés que d'aspects gratifiants. Parmi les difficultés, elles ont souligné des caractéristiques problématiques de l'enfant, les attitudes et relations avec l'entourage, l'accès aux services et l'impact sur la vie personnelle, sociale ou de couple. Elles ont identifié quelques gratifications, dont certaines caractéristiques positives de l'enfant, son progrès malgré le DAH et la croissance personnelle de la mère. Les conjoints et les grands-parents fournissent du soutien tangible, les amis et les autres mères vivant avec le DAH fournissent du soutien informationnel, et les sources de soutien affectif sont multiples. Les sources formelles (professionnelles, scolaires, communautaires et groupes d'entraide) fournissent de l'aide sous forme de collaboration, dépistage, adaptation, compréhension et références. Cependant, certaines de ces sources d'aide peuvent aussi amener des difficultés. Les mères rencontrées avaient besoin de plus de soutien formel en français, particulièrement du répit et des groupes d'entraide. Une sensibilisation auprès de la communauté s'impose, étant donné la persistance des attitudes négatives.

\section{Les jeunes : une réalité ambiguë et contradictoire}

\section{Tania Duguay}

École de service social, Université d'Ottawa

La jeunesse est un "phénomène » incertain. Groupe social ambigu, on ne sait pas comment le définir et quelle place lui accorder 
dans la société contemporaine. Les jeunes sont une création sociale reflétant les diverses idéologies de la société. Aujourd'hui, la jeunesse est perçue de plus en plus comme un problème social et elle devient très vite une cible pour les politiques sociales. Dès le début du $\mathrm{XX}^{\mathrm{e}}$ siècle, il se développe un système spécifique pour mineurs, qui vient encadrer les jeunes dits victimes de leur milieu, ainsi que les jeunes dits déviants et dangereux pour la société. Les jeunes qui sont étiquetés "victimes " tombent sous le système de protection et ceux étant étiquetés « déviants » sont pris en charge par le système pénal. Les jeunes sont souvent perçus comme faisant partie d'un phénomène "blanc " ou "noir ». Ceux qui n'entrent dans aucune de ces deux définitions se retrouvent en marge du système, dans le "no mans land ». Depuis près d'un siècle, les jeunes sont maintenus dans des situations vulnérables à cause de façons dont on les définit et de la mise en place des politiques sociales. Ces mêmes politiques viennent justifier l'opinion publique expliquant que les jeunes forment évidemment une population dangereuse. Alors, leur place est de plus en plus réduite dans la société actuelle par la tentative de mise en place d'un système pour mineurs.

\section{Motifs du non-retour aux études chez des jeunes n'ayant pas terminé leur secondaire: recherche exploratoire}

Tania Paiement

École de service social, Université d'Ottawa

Dans le contexte social actuel où l'instruction est considérée comme un pilier de la société, nous pouvons nous questionner sur le sort réservé aux adolescents qui quittent l'école secondaire avant d'avoir obtenu leur diplôme. Les raisons du départ précoce, souvent évoquées dans les écrits, sont fort complexes et touchent 
autant les aspects personnels, socioéconomiques que scolaires. La trajectoire des jeunes adultes suite au départ scolaire peut être décrite en grande partie par la précarité de l'emploi et le recours aux services d'assurance-emploi et d'assistance sociale. En effet, les sortants scolaires éprouvent de façon générale plus de difficulté à percer le marché du travail que les jeunes plus scolarisés. Cela étant dit, quelles raisons incitent les sortants scolaires du secondaire à ne pas retourner aux études afin d'obtenir leur diplôme? Ce mémoire tente donc de répondre à cette question : pourquoi les sortants scolaires ne cherchent-ils pas à retourner aux études afin d'obtenir leur diplôme d'études secondaires (DES) et d'améliorer leurs possibilités d'embauche?

Afin de répondre à cette question, nous avons interviewé individuellement cinq sortants scolaires. Tous les sortants ont quitté l'école en 1997. Deux filles et trois garçons, dont le niveau de scolarité variait entre la $2^{\text {ième }}$ et la $4^{\text {ième }}$ année du secondaire, ont participé à ces entretiens qui tentaient de reconstruire la période de quatre ans s'étant écoulée depuis leur départ scolaire et le moment de l'entrevue.

Les analyses, bien que limitées à cause du petit échantillon, ont permis d'établir trois hypothèses pour la suite des recherches. D'abord, les responsabilités financières et les obligations familiales peuvent jouer un rôle déterminant dans l'intention de retourner aux études. Deuxièmement, les représentations que se font les jeunes à l'égard de l'institution scolaire peuvent avoir des incidences sur les intentions de retour scolaire et finalement, le retour scolaire ne représente pas toujours la meilleure solution pour les sortants.

De plus, les résultats nous ont permis de suggérer trois implications pour l'intervention : un programme de financement pour les personnes faiblement salariées occupant des emplois précaires; une campagne de visibilité pour les organismes d'aide à l'insertion et enfin, la valorisation et la reconnaissance des formations professionnelles. 


\title{
L'autonomie chez la personne âgée en institution : mythes ou réalités
}

\author{
Thomas Vanté \\ École de service social, Université d'Ottawa
}

La notion d'autonomie utilisée dans le domaine du vieillissement se caractérise par une connotation négative. Elle s'inscrit généralement dans un modèle déficitaire, c'est-à-dire qu'elle est surtout définie et présentée sous l'angle de sa perte. En effet, la majorité des écrits sur la notion d'autonomie chez les personnes âgées en institution les présentent comme des personnes avec des pertes de capacités physiques et cognitives importantes. Cependant, n'y a-t-il pas une autre façon de parler de l'autonomie chez cette clientèle? Si oui, quel est le sens donné à cette autonomie? Telles sont les interrogations auxquelles ce mémoire cherche à répondre.

Pour ce faire, nous voulons présenter une perspective qui aborde la question de l'autonomie des personnes âgées sous un angle plus éthique et favorable à cette clientèle. Il s'agit de la perspective fondamentale de l'autonomie telle qu'élaborée par Zuniga et Boucher (1993). Cette perspective voit la personne âgée comme une personne à part entière en dépit de certaines pertes physiques ou cognitives. Ainsi, c'est sur elle que s'appuie notre objet d'étude.

Donc, le but de ce mémoire consiste à jeter un regard nouveau sur la notion d'autonomie à partir d'une approche qui voit la personne âgée dans son entité. Plus particulièrement, le présent mémoire se propose d'explorer la mesure dans laquelle l'approche fondamentale de l'autonomie pourrait contribuer à une meilleure compréhension de l'autonomie chez les personnes âgées en institution et permettre, par le fait même, une meilleure intervention.

Ce mémoire se divise en quatre parties. La première section élabore la problématique de l'autonomie chez la personne âgée. 
Nous y décrivons la construction de la notion d'autonomie à travers le temps en plus de présenter le cadre théorique privilégié et la question de recherche. La deuxième section présente la méthodologie sur laquelle repose notre travail, soit une analyse critique de la littérature portant sur la notion d'autonomie chez les personnes âgées. Nous présentons ensuite l'analyse de la documentation. Le tout se termine par une réflexion sur les implications pour l'intervention en service social auprès des personnes âgées en institution.

\section{La thérapie brève orientée vers les solutions : sa description et ses applications}

\section{Tina Delorme}

Université d'Ottawa, École de service social

Dans leur milieu de travail, les travailleuses et les travailleurs sociaux sont parfois appelés à pratiquer de la thérapie familiale ou individuelle. Malgré la diversité de modèles d'intervention accessibles, les approches brèves sont en voie d'expansion depuis les dernières années. Lors de notre stage en service social, nous avons pu observer la mise en pratique d'un modèle de traitement bref, la thérapie brève orientée vers les solutions (Solution-Focused BriefTherapy). À la suite de nos observations, nous avons décidé d'approfondir nos connaissances sur ce modèle d'intervention. Dans cette recherche, nous avons tenté de décrire ce qu'est la thérapie brève orientée vers les solutions, comment et dans quelles circonstances elle est mise en pratique. Afin de répondre à ces questions, nous avons exploré l'historique et le contexte sociopolitique des traitements brefs, ainsi que les origines de cette forme de thérapie. Nous avons identifié les principes qui la régissent, les moyens et les techniques qui lui sont propres. Par la suite, nous avons exploré l'application de la thérapie dans des contextes de problématiques lourdes et sévères, plus précisément, dans les 
domaines de la psychiatrie, de la violence, de la protection de l'enfant ainsi qu'en contexte d'autorité. En comparant ses applications dans des circonstances variées, nous avons relevé les points communs, ou similarités, ainsi que les différentes modalités de sa mise en pratique adaptées aux situations variées. Enfin, nous avons tenté de faire ressortir les points forts et les faiblesses de la thérapie brève orientée vers les solutions. Nous suggérons, par conséquent, la poursuite de plus amples recherches visant à établir la validité des résultats obtenus à la suite de l'application de ce modèle thérapeutique. Il y a place pour des études plus approfondies et des études comparatives dans les différents champs d'application. Enfin, nous souhaitons et encourageons la production plus abondante d'une documentation en français. 